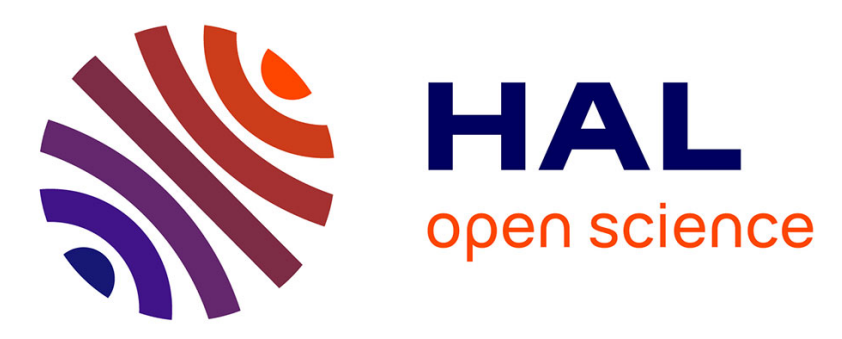

\title{
High aspect ratio nanochannel machining using single shot femtosecond Bessel beams
}

M.K. Bhuyan, F. Courvoisier, P.-A. Lacourt, M. Jacquot, R. Salut, L. Furfaro, J.M. Dudley

\section{- To cite this version:}

M.K. Bhuyan, F. Courvoisier, P.-A. Lacourt, M. Jacquot, R. Salut, et al.. High aspect ratio nanochannel machining using single shot femtosecond Bessel beams. Applied Physics Letters, 2010, 97 (8), pp.081102. 10.1063/1.3479419 . hal-00517173

\section{HAL Id: hal-00517173 \\ https://hal.science/hal-00517173}

Submitted on 3 May 2021

HAL is a multi-disciplinary open access archive for the deposit and dissemination of scientific research documents, whether they are published or not. The documents may come from teaching and research institutions in France or abroad, or from public or private research centers.
L'archive ouverte pluridisciplinaire HAL, est destinée au dépôt et à la diffusion de documents scientifiques de niveau recherche, publiés ou non, émanant des établissements d'enseignement et de recherche français ou étrangers, des laboratoires publics ou privés. 


\title{
High aspect ratio nanochannel machining using single shot femtosecond Bessel beams
}

\author{
M. K. Bhuyan, F. Courvoisier, ${ }^{\text {a) }}$ P. A. Lacourt, M. Jacquot, R. Salut, L. Furfaro, and \\ J. M. Dudley \\ Département d'Optique P. M. Duffieux, Institut FEMTO-ST, UMR 6174, CNRS-Université de \\ Franche-Comté, 16 route de Gray, 25030 Besançon Cedex, France
}

(Received 18 June 2010; accepted 23 July 2010; published online 23 August 2010)

\begin{abstract}
We report high aspect ratio nanochannel fabrication in glass using single-shot femtosecond Bessel beams of sub-3 $\mu \mathrm{J}$ pulse energies at $800 \mathrm{~nm}$. We obtain near-parallel nanochannels with diameters in the range $200-800 \mathrm{~nm}$, and aspect ratios that can exceed 100. An array of $230 \mathrm{~nm}$ diameter channels with $1.6 \mu \mathrm{m}$ pitch illustrates the reproducibility of this approach and the potential for writing periodic structures. We also report proof-of-principle machining of a through-channel of $400 \mathrm{~nm}$ diameter in a $43 \mu \mathrm{m}$ thick membrane. These results represent a significant advance of femtosecond laser ablation technology into the nanometric regime. (C) 2010 American Institute of Physics. [doi:10.1063/1.3479419]
\end{abstract}

Femtosecond (fs) laser machining of dielectrics has found wide application, from waveguide writing to the fabrication of nanometer scale structures. ${ }^{1}$ A particular challenge, however, is fabricating high aspect ratio channels of submicron transverse dimensions, because strong focusing of Gaussian beams typically limits the longitudinal machining region to only $1 \mu \mathrm{m} .{ }^{1,2}$ Although modified Gaussian beam geometries have demonstrated submicron channels over depths $\sim 10 \mu \mathrm{m}$, these setups lead to strongly interdepen- dent diameter and channel length, and show irregular structure due to nonlinear beam distortion. ${ }^{3}$ In this paper, we report fs nanochannel machining using diffraction-free Bessel beams to achieve uniform energy deposition over extended lengths. ${ }^{4-6}$ We perform single-shot experiments fabricating nanochannels in glass with subwavelength diameters in the range $200-800 \mathrm{~nm}$, and lengths up to $30 \mu \mathrm{m}$. We also report proof-of-principle through-channel fabrication. We study the effect of Bessel beam conical angle and pulse energy, and (a)

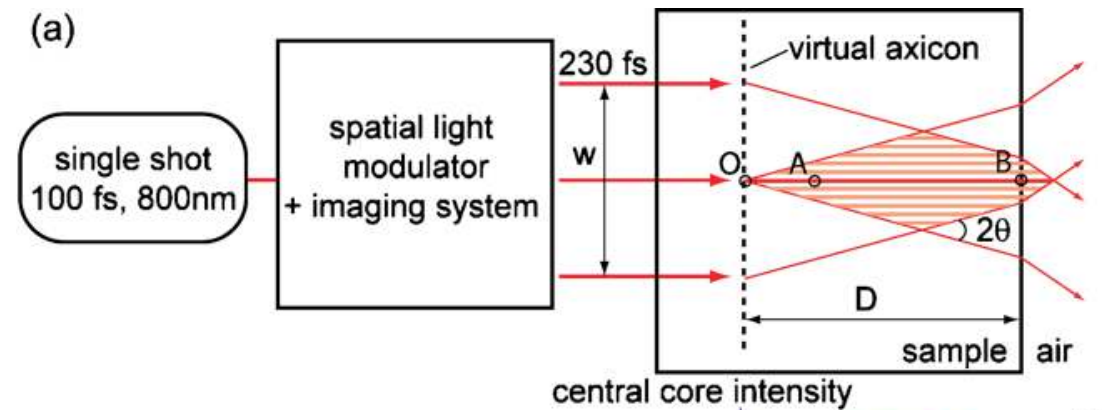

central core intensity

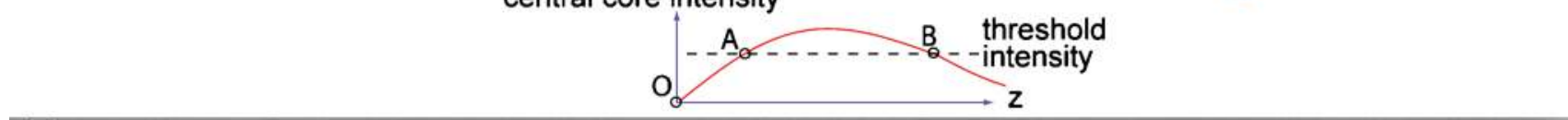

(b)

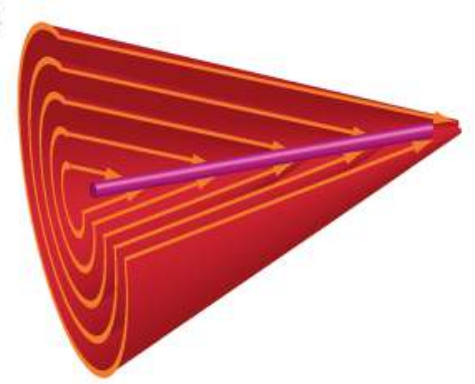

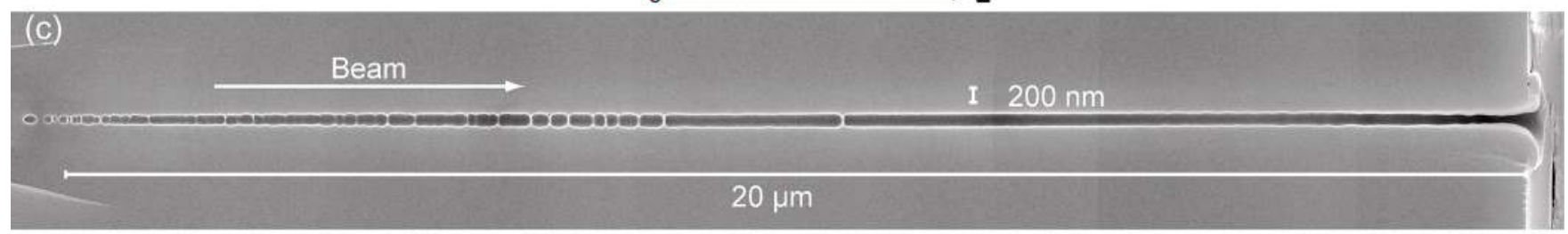

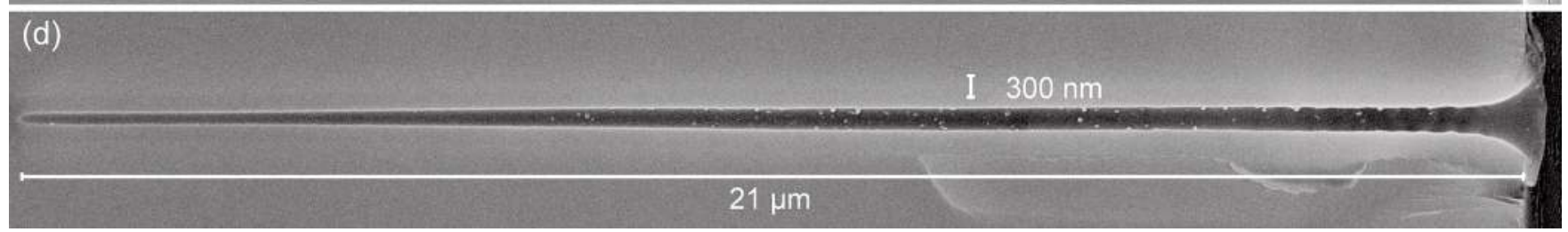

FIG. 1. (Color online) (a) Experimental setup. The curve shown below the sample illustrates the variation of the core intensity along the Bessel beam. Ablation occurs over a range between points A and B. (b) Representation of the conical energy flux toward the central core. (c) and (d) show terminated channels for energies of $0.65 \mu \mathrm{J}$ and $0.85 \mu \mathrm{J}$, respectively.

${ }^{a)}$ Electronic mail: francois.courvoisier@femto-st.fr. 


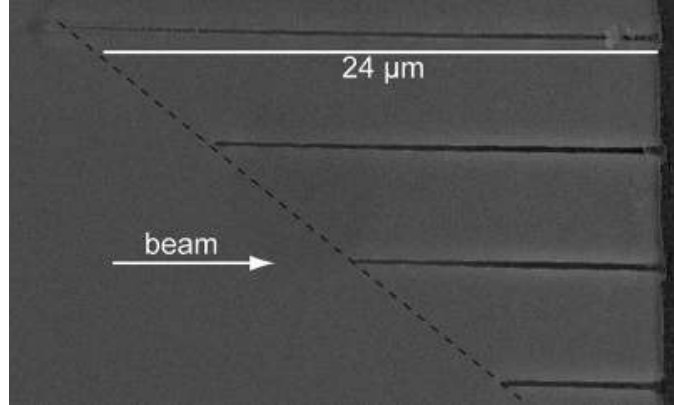

FIG. 2. SEM image showing four machined channels with sample displacement differing by $4 \mu \mathrm{m}$ in the horizontal direction and $5 \mu \mathrm{m}$ vertically. The relative error in channel length due to cleaving is $\pm 0.5 \mu \mathrm{m}$.

demonstrate independent control of channel length and diameter. Although Bessel beams have been previously applied to surface ablation and micrometer scale channels in a multishot regime, ${ }^{7,8}$ our results significantly extend this state-ofthe-art to nanoscale dimensions.

Ideal zero order Bessel beams are formed from the axially-symmetric interference of two plane waves to yield a high intensity central core surrounded by lower intensity concentric rings. ${ }^{4}$ Bessel beams are "diffraction-free," maintaining near constant profile over distances far larger than the Rayleigh length of Gaussian beams, and they can exhibit stationary propagation even in the presence of nonlinear losses. ${ }^{5}$ Indeed, although Gaussian beams typically undergo deleterious nonlinear beam distortion at ablation-level intensities, Bessel beams can resist such instabilities, allowing for uniform energy deposition over extended propagation lengths. ${ }^{6}$

Our experiments use the setup shown in Fig. 1(a). ${ }^{7}$ Using an amplified Ti:Sapphire laser system at $800 \mathrm{~nm}$, we synthesize the Bessel beam using a programmable phase mask to generate a "virtual axicon," defining the beam onset (point $\mathrm{O}$ in Fig. 1). Our setup allows the beam onset to be placed at any required position, including within a sample. The pulse duration at the imaging system output was $\sim 230 \mathrm{fs}$, and we use axially symmetric polarization. Figure 1(b) shows how the conical energy flux from the interfering fields is directed toward the central core. All experiments here used single shot illumination and were carried out in Corning 0211 borosilicate glass at two values of conical half angle $\theta$ in glass: $17^{\circ}$ and $11^{\circ}$. After processing a series of channels for a given set of parameters, microtrench laser machining outside the region of interest induced a stress plane at a small angle with respect to the series, allowing cleaving with $\pm 50 \mathrm{~nm}$ precision through one channel for scanning electron microscopy (SEM) imaging.

We investigated terminated channel machining for pulse energies of 0.2-2.5 $\mu \mathrm{J}$. Figures 1(c) and 1(d) show SEM images of channels machined for conical half angle $\theta_{1}=17^{\circ}$. Here, the Bessel beam central lobe has $660 \mathrm{~nm}$ diameter at half maximum and longitudinal extent of $30 \mu \mathrm{m}$ at half maximum in glass. Provided that the intensity in the central lobe at the exit surface (point $B$ in Fig. 1) exceeded the optical breakdown threshold $I_{\text {th }}$, a nanochannel was formed between the exit face and the position within the sample where the intensity of the central lobe first exceeds $I_{\text {th }}$ (point A in Fig. 1). The minimal energy where we observed a clear contiguous channel structure was $\sim 0.7 \mu \mathrm{J}$, corresponding to a maximal peak intensity of $1.1 \times 10^{14} \mathrm{~W} / \mathrm{cm}^{2}$. Figure 1 shows two channels machined on either side of this point with energies of 0.65 [Fig. 1(c)] and $0.85 \mu \mathrm{J}$ [Fig. 1(d)] for a distance from beam onset to exit face $D=26 \mu \mathrm{m}$. In both cases we clearly see extended channels over $20 \mu \mathrm{m}$ lengths with mean diameters of 200 and $330 \mathrm{~nm}$ but with a lower energy of $0.65 \mu \mathrm{J}$, we see transverse bridges across the channel which we attribute to resolidification during ablated material expansion and evacuation. We also note that the exit surfaces show a hemitorus of resolidified debris. The channel aspect ratios are, respectively, 100 (c) and 60 (d).

Channel length is varied straightforwardly by changing the beam onset position in the sample using a motorized translation stage. Figure 2 shows four channels machined at $0.73 \mu \mathrm{J}$ pulse energy, where the sample-beam displacement was in $\Delta \mathrm{x}=4 \mu \mathrm{m}$ steps. This varies the longitudinal position at which the optical breakdown threshold is reached and modifies the channel length. Indeed, we see a reduction in machined channel length of $\Delta \mathrm{l}=\mathrm{n} \Delta \mathrm{x} \sim 6 \mu \mathrm{m}$ between steps, where $n=1.51$ is the sample refractive index. Significantly, the mean channel diameter $(250 \mathrm{~nm})$ is invariant with the beam onset position, demonstrating the ability to decouple the channel diameter and length. We also carried out experiments with both breakdown threshold points (A, B) entirely within the $150 \mu \mathrm{m}$ thick sample. However, with this geometry, we did not see evidence for enclosed void formation ${ }^{9}$ although refractive index modification was observed. This highlights the need to ensure a breakdown point at an exit surface to facilitate material evacuation. Indeed, using a thinner $43 \mu \mathrm{m}$ thick membrane, we demonstrated proof-ofprinciple through-channel machining under conditions where the beam intensity exceeded breakdown threshold at both input and exit faces. Figure 3 shows results using a conical half angle $\theta_{2}=11^{\circ}$ and energy per pulse $3.1 \mu \mathrm{J}$ with the beam position centered in the membrane. The channel is open to both sides of the membrane, although uniformity is reduced compared to the terminated channels. In this context, however, we note that the nonlinear stationarity of Bessel beams can depend sensitively on interface effects, ${ }^{6}$ and optimizing through channel fabrication will require detailed studies of the role of effects such as nonlinear stability and surface field enhancement.

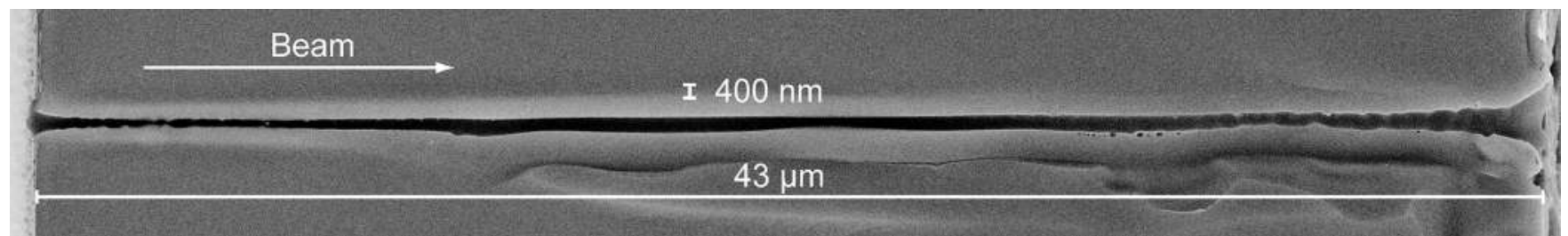

FIG. 3. Proof of principle machining of a through channel using Bessel beam conical half angle $\theta_{2}=11^{\circ}$ and a $43 \mu \mathrm{m}$ thick glass membrane at single shot pulse energy of $3.1 \mu \mathrm{J}$. 


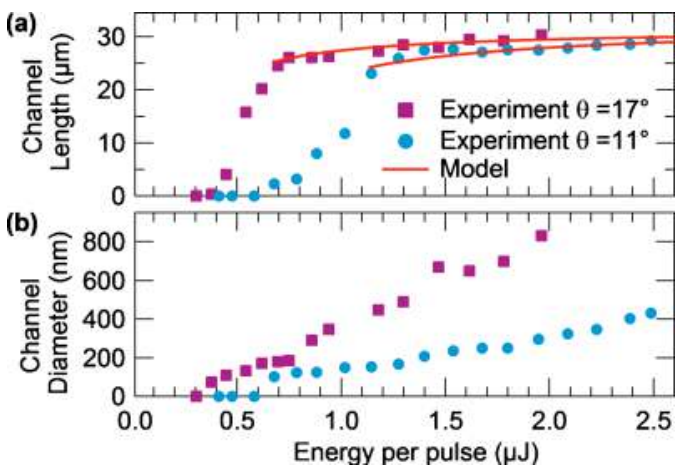

FIG. 4. (Color online) Dependence on pulse energy of (a) length and (b) mean diameter of machined nanochannel for two different conical half angles $\theta_{1}=17^{\circ}$ (squares) and $\theta_{2}=11^{\circ}$ (circles). Due to cleaving precision, the error bars are $\pm 2 \mu \mathrm{m}$ for channel length and $\pm 50 \mathrm{~nm}$ for mean channel diameter.

We also investigated the dependence of the terminated channel characteristics on pulse energy and conical angle. Figure 4 shows results for an energy range $0.2-2.5 \mu \mathrm{J}$ and for conical angles of $\theta_{1}=17^{\circ}$ and $\theta_{2}=11^{\circ}$ (central lobe diameters, respectively, $660 \mathrm{~nm}$ and $1.01 \mu \mathrm{m}$ ). In both cases, the beam onset was positioned at $D \sim 32 \mu \mathrm{m}$. Although the intensity distribution in the sample varies for each case, the central lobe intensity at the output face is identical. Channel length and diameter were determined from SEM images. Figure 4(a) shows the variation in channel length with pulse energy. After a transition regime where the length of the channels rapidly increases with energy, it slowly tends to an asymptotic value given by $D$ (the distance OB from the virtual axicon to the exit face). Transverse bridges of resolidified material are observed only in the transition regime and in this regime, we measured the channel length from the surface to the point where the damage regions separated by the bridges became noncontiguous. Above this regime, the measured channel lengths agree quantitatively with calculations of the longitudinal extent of the beam profile where the intensity exceeds $I_{\mathrm{th}}$. Writing the on-axis intensity at a propagation length $z$ from the beam onset as: ${ }^{10} I(z)$ $=8 \pi P_{0} n z \sin ^{2} \theta /\left(\lambda w^{2}\right) \exp \left[-2(z \sin \theta / w)^{2}\right]$, the red curves in Fig. 4(a) show the calculated range of $z$ over which optical breakdown is reached, from point A in Fig. 1 to the sample exit side. Here, $w$ is the waist of the initial Gaussian beam, $P_{0}$ is the input peak power and we used a threshold intensity of $I_{\mathrm{th}}=5.5 \times 10^{13} \mathrm{~W} / \mathrm{cm}^{2}$, within the range reported for borosilicate glass. ${ }^{1}$ Although this model would not be expected to describe the channel properties in the transition regime when there are resolidification effects, it accurately describes terminated channel length at higher energies.

Figure 4(b) shows the energy-dependence of channel diameter. We note an approximately linear dependence above the transition regime, and we see that the channel diameters for $\theta_{1}=17^{\circ}$ are greater than for $\theta_{2}=11^{\circ}$. This dependence can be readily understood since a larger conical angle yields a smaller central lobe diameter and thus a higher energy density, and it is the energy density that primarily influences the

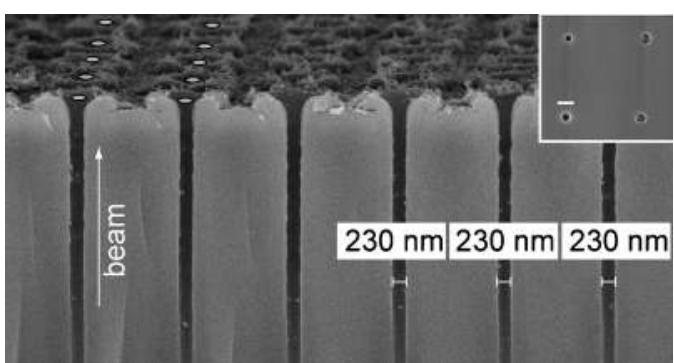

FIG. 5. Profile of an array of channels drilled with identical $0.70 \mu \mathrm{J}$ energy per pulse and beam position such that channel length is $10 \mu \mathrm{m}$ and diameter $230 \mathrm{~nm}$. The pitch is $1.6 \mu \mathrm{m}$. White circles indicate channel positions. Inset shows postprocessed surface for an additional sample closer to threshold with pitch $800 \mathrm{~nm}$. Scale bar is $200 \mathrm{~nm}$.

hydrodynamic material removal. ${ }^{9}$ Above the transition regime, the process is highly reproducible. We demonstrate this explicitly by writing a $32 \times 72 \mu \mathrm{m}^{2}$ array of nanochannels with $\theta_{1}=17^{\circ}, 0.70 \mu \mathrm{J} /$ pulse and a pitch of $1.6 \mu \mathrm{m}$. A SEM image from the center of the machined region is shown in Fig. 5. The high shot-to-shot reproducibility is immediately apparent and we measure only $<7 \%$ difference in mean channel diameter $(230 \mathrm{~nm})$. We determined that the minimal achievable distance between adjacent channels with no structural deformation was $\sim 600 \mathrm{~nm}$ for parameters as above. Residual debris on the surface can be readily removed using focused ion beam postprocessing (see inset).

In conclusion, our results demonstrate the fabrication of high aspect ratio nanochannels in glass using single-shot fs Bessel beams. The channel wall parallelism is attributed to the fundamental stationarity of Bessel beams which allows them to resist transverse beam breakup at ablation-level intensities. Our results represent an important application of fs laser nanoprocessing, which is applicable to all dielectric materials and allows for independent control of channel length and diameter. We anticipate wide application to nanofluidics and nanophotonics.

We acknowledge the Région Franche-Comté and the Institut Universitaire de France for funding.

${ }^{1}$ R. R. Gattass and E. Mazur, Nat. Photonics 2, 219 (2008).

${ }^{2}$ S. I. Kudryashov, G. Mourou, A. Joglekar, J. F. Herbstman, and A. J. Hunt, Appl. Phys. Lett. 91, 141111 (2007).

${ }^{3}$ Y. V. White, X. Li, Z. Sikorski, L. M. Davis, and W. Hofmeister, Opt. Express 16, 14411 (2008).

${ }^{4}$ J. Durnin, J. J. Miceli, and J. H. Eberly, Phys. Rev. Lett. 58, 1499 (1987).

${ }^{5}$ M. A. Porras, A. Parola, D. Faccio, A. Dubietis, and P. Di Trapani, Phys. Rev. Lett. 93, 153902 (2004).

${ }^{6}$ P. Polesana, M. Franco, A. Couairon, D. Faccio, and P. Di Trapani, Phys. Rev. A 77, 043814 (2008)

${ }^{7}$ F. Courvoisier, P.-A. Lacourt, M. Jacquot, M. K. Bhuyan, L. Furfaro, and J. M. Dudley, Opt. Lett. 34, 3163 (2009).

${ }^{8}$ M. K. Bhuyan, F. Courvoisier, P.-A. Lacourt, M. Jacquot, L. Furfaro, M. J. Withford, and J. M. Dudley, Opt. Express 18, 566 (2010).

${ }^{9}$ S. Juodkazis, K. Nishimura, S. Tanaka, H. Misawa, E. G. Gamaly, B. Luther-Davies, L. Hallo, P. Nicolai, and V. T. Tikhonchuk, Phys. Rev. Lett. 96, 166101 (2006).

${ }^{10}$ V. Jarutis, R. Paškauskas, and A. Stabinis, Opt. Commun. 184, 105 (2000). 